Name wird als Facharzt sowohl in Bezug auf die B-Klinik wie aber auch die Privatpraxis ausdrücklich genannt. Für den unbefangenen Betrachter ergibt sich aus der „Registrierung als Facharzt" eine Zusammenarbeit der benannten Ärzte mit dem Versicherungsunternehmen. Tatsächlich besteht auch ausweislich der als Anlage 6 überreichten Ausführungen der Firma $\mathrm{N}$ eine geschäftliche Beziehung jedenfalls insoweit, als „,mit der Empfehlung von N“ dem Arzt nicht nur ein weiteres Argument für den Verkauf medizinischer Leistungen an die Hand gegeben wird, sondern unter Arztsuche auch ,für Sie und ihre Praxis/Klinik eine kostenlose Werbeplattform und Präsentationsmöglichkeit" entsteht.

[23] Damit erweist sich jedenfalls für den hier aufgrund der Antragsfassung allein relevanten Bereich kumulativen Zusammentreffens von konkreter Werbung für $\mathrm{N}$ und $\mathrm{Re}-$ gistrierung als Facharzt der Umstand des Listenlassens als unlauter, ohne dass es darauf ankommt, ob auch weitere gelistete Ärzte sich in gleicher Weise geschäftlich unlauter verhalten.

[24] Ob das Verhalten des Bekl. zugleich gegen $₫ 31$ BOÄ verstößt bedarf einer Entscheidung.

[25] Neben der Unterlassung schuldet der Bekl. gem. \12 Abs. 1 S. 2 UWG die Erstattung der in ihrer Höhe nicht streitigen Abmahnkosten. Der Betrag von 246,10€ ist antragsgemäß ab dem 23.3.2016 wegen Verzuges zu verzinsen.

DOI: $10.1007 / \mathrm{s} 00350-017-4637-6$

\section{Anmerkung zu OLG Celle, Beschl. v. 2.5.2016 - 13 U 155/15 (n. rechtskr.) und zu LG Düsseldorf, Urt. v. 19.8.2016 - 380 15/16}

\section{Hans-Dieter Lippert}

Berufsrechtliche Vorschriften genießen gemeinhin einen eher geringen Bekanntheitsgrad, der auch mit ihrer Beachtung korreliert. Warum die jeweiligen Berufsträger sich so wenig darum kümmern, lässt sich schwer erklären. Ihre Nichtbeachtung kann dennoch gravierende Folgen nach sich ziehen, wie die beiden vorstehend abgedruckten Entscheidungen zeigen. Beide Entscheidungen sind zu $\$ 3$ BOÄ, also zur Berufsordnung für Ärzte ergangen. Die Brisanz des Verstoßes gegen $\$ 3$ BOÄ der Entscheidungen liegt aber erst aus der Fernwirkung des UWG, also dem unlauteren Verhalten der betreffenden Ärzte im Geschäftsverkehr. Dieses folgt aus der Missachtung von $\$ 3$ BOÄ.

Der Schutzzweck von $\$ 3$ BOÄ besteht darin, dass der Arzt in Diagnose und Therapie seine (ärztliche) Unabhängigkeit bewahren soll und sich nicht in einer Weise gewerblich betätigt, die dem vertrauensvollen Arzt-Patienten-Verhältnis zuwider läuft ${ }^{1}$. Dazu gehört es auch, dass er nicht mit Namen und Titel in gewerblicher Weise wirbt oder damit werben lässt. Geschieht die Werbung im Zusammenhang mit Arzneimitteln oder Medizinprodukten, dann präzisiert $\$ 11 \mathrm{HWG}$ dieses Verbot noch im Einzelnen.

Abgesehen von dieser eher branchenspezifischen Regelung im HWG ist auf das Handeln der Ärzte im Geschäftsverkehr auch das UWG anwendbar. Hier ist es vor allem der Verstoß gegen $\$ 3$ a UWG, also das unlautere Verhalten durch Zuwiderhandeln gegen eine Vorschrift, die den Schutz der Marktteilnehmer aber auch des Verbrauchers zum Ziel hat und bei der der Verstoß deren Interessen spürbar beeinträchtigen kann. Es ist dies der $₫ 4 \mathrm{Nr}$. $11 \mathrm{UWG}$ in seiner alten Fassung, der hier zur Anwendung kommt. Bei $\$ 3$ Abs. 1 BOÄ handelt es sich um eine Norm, bei der der

Rechtsanwalt Dr. iur. Hans-Dieter Lippert,

KNORR Rechtsanwälte,

Frauenstraße 11, 89073 Ulm, Deutschland
Verstoß den Patienten, also einen Verbraucher beeinträchtigen kann ${ }^{2}$. Auch die Health Claims - VO $(\mathrm{HCV})^{3}$ enthält, wie die Entscheidung des OLG Celle zeigt, Verbote, die sich im Bereich des UWG als unlauter erweisen.

In diesem Fall ging es um eine Werbung für Lebensmittel (NEM) und ausnahmsweise einmal nicht um Arzneimittel oder Medizinprodukte. Dennoch zeigt der Fall, dass die Anzahl einschlägiger Vorschriften, die das Verbot des $\$ 3$ BOÄ konkretisieren können, zahlreicher geworden sind.

Im Fall des LG Düsseldorf musste nicht so weit ausgeholt werden. Das Verhalten des Arztes als das eines Versicherungsvertreters oder auch eines Kreditvermittlers ist bereits als ein Verstoß gegen $\$ 3$ BOÄ zu werten und damit unlauter. Das Sich-Verstecken hinter einer juristischen Person $(=\mathrm{GmbH})$, hat das Gericht ebenfalls zu Recht nicht akzeptiert.

Bleibt in beiden Fällen die spannende Frage, wie die jeweilig zuständige Ärztekammer auf diese Verstöße reagieren wird. Hoffentlich waltet hier nicht zuviel kollegiale Nachsicht. Nachahmer würde dies geradezu animieren.

1) So oder ähnlich auch Ratzel, in: ders./Lippert, MBÖ̈, 6. Aufl 2015, 3 3, Rdnr. 1, Scholz, in: Spickhoff, Medizinrecht, 2. Aufl. 2014, \$3 MBO, Rdnr. 1, Rehborn, in: Prütting, Medizinrecht, 2. Aufl. 2015, MBO \&3, Rdnr. 2.

2) So Köhler, in: ders./Bornkamm, UWG, 35. Aufl. 2017, 『3a Rdnr. 1.132; Ohly/Sosnitza, UWG, 7. Aufl. 2016, §3a, Rdnr. 53 jeweils m. w. Nachw.

3) VO (EG) Nr. 1924/2006 des Europäischen Parlaments und des Rates vom 20.12.2006 über nährwert- und gesundheitsbezogene Angaben über Lebensmittel, Abl. L 404 v. 30.12.2006, S. 9.

\section{Übertragung von Geschäftsanteilen an einer MVZ-GmbH und Wirksamkeit von Treuhandverträgen}

EulMSVO Art. 3 Abs. 1; BGB §134; SGB V §§95 Abs. 1a S. 1, 126 Abs. 3

1. Die Veräußerung und Übertragung von Geschäftsanteilen einer als MVZ zugelassenen GmbH an Dritte, die nicht über die Gründereigenschaft nach $\$ 95$ Abs. 1a SGB V verfügen, ist wegen Verstoß gegen ein gesetzliches Verbot nichtig.

2. Entsprechende Treuhandvereinbarungen stellen ein unwirksames Umgehungsgeschäft dar und unterliegen der Nichtigkeitsfolge des $\$ 134$ BGB. (Leitsätze des Bearbeiters)

LG Köln, Urt. v. 1.12.2016 - 5 O 236/15

Problemstellung: Das Urt. des LG Köln befasst sich, soweit ersichtlich, erstmals mit dem Anwendungsbereich des $\$ 95$ Abs. 1a S. 1 SGB V i.d.F. GKV-VStG v. 22.12.2011 (dazu Bäune/Dahm/Flasbarth, MedR 2012, 77 ff.) und der durch das Gesetz erfolgten Neuregelung der Gründungsvoraussetzungen für Medizinische Versorgungszentren (MVZ). Veranlasst war die Entscheidung durch Veräußerung und Übertragung von Geschäftsanteilen an die Beklagten in Zusammenhang mit der Einleitung eines Insolvenzverfahrens sowie den Abschluss von Treuhandverträgen anlässlich der Rückübertragung der Gesellschaftsanteile.

Eingesandt von Dr. iur. Sebastian Gall, Frankfurt; bearbeitet von Rechtsanwalt und Notar Prof. Dr. iur. Franz-Josef Dahm,

Fachanwalt für Verwaltungsrecht und Fachanwalt für Medizinrecht, Sozietät Schmidt, von der Osten \& Huber PartmbB,

Haumannplatz 28, 45130 Essen, Deutschland 